\title{
Acidentes domésticos em idosos atendidos em um hospital de urgência
}

Ana Maria Ribeiro dos Santos ${ }^{1}$, Dáfne Beatriz Dias Pereira ${ }^{2}$, Lays Carollinne Soares de Carvalho ${ }^{3}$, Maria Zélia de Araújo Madeira ${ }^{4}$, Elaine Maria Leite Rangel Andrade 5

\footnotetext{
${ }^{1}$ Enfermeira, Doutora em Enfermagem. Professora Adjunto da Universidade Federal do Piauí. Teresina, PI, Brasil. E-mail: ana.mrsantos@gmail.com.

${ }^{2}$ Enfermeira. Teresina, PI, Brasil. E-mail: dafnebeatrizdias@gmail.com.

${ }^{3}$ Enfermeira. Teresina, PI, Brasil. E-mail: layscarollinne@hotmail.com.

${ }^{4}$ Enfermeira, Doutora em Ciências Médicas. Professora Adjunto da Universidade Federal do Piauí. Teresina, PI, Brasil. E-mail:

zeliamadeira15@yahoo.com.br.

${ }^{5}$ Enfermeira, Doutora em Enfermagem. Professora Adjunto da Universidade Federal do Piauí. Teresina, PI, Brasil. E-mail: elairgel@gmail.com.
}

Recebido: 04/07/2015.

Aceito: 11/03/2016.

Publicado: 30/06/2016.

Como citar esse artigo:

Santos AMR, Pereira DBD, Carvalho LCS, Madeira MZA, Andrade EMLR. Acidentes domésticos em idosos atendidos em um hospital de urgência. Rev. Eletr. Enf. [Internet]. 2016 [acesso em:

____;18:e1169. Disponível em: http://dx.doi.org/10.5216/ree.v18.36569.

\section{RESUMO}

Estudo transversal realizado em hospital de referência em urgência com amostragem por conveniência cujos dados foram coletados de junho a julho de 2014. Objetivou analisar os acidentes domésticos em idosos atendidos em hospital público de urgência. Do total de 83 entrevistados, $77,1 \%$ eram mulheres, possuíam 80 a 89 anos (32,5\%), analfabetos $(32,5 \%)$, viúvos $(44,6 \%)$, residindo com cônjuge e outros familiares $(73,5 \%)$, aposentados $(84,4 \%)$, recebiam um a dois salários mínimos (88\%). A prevalência de acidentes domésticos foi de 6,4\%, com predominância de queda $(84,4 \%)$, da própria altura $(55,6 \%)$. Descuido na observação do ambiente foi auto referido como principal causa (45,8\%). A não associação entre a ocorrência de quedas, existência de doença crônica, medicação de uso contínuo e realização de atividade no instante do acidente foi um achado relevante. Recomenda-se cuidado multiprofissional com avaliação funcional ampla identificando as deficiências dos idosos e limitações ambientais visando orientação comunitária e planejamento de intervenções integradas.

Descritores: Acidentes Domésticos; Idoso; Ferimentos e Lesões; Enfermagem Geriátrica.

\section{INTRODUÇÃO}

A longevidade humana, fenômeno comum à maioria dos países, está associada à transição demográfica, caracterizada por uma sequência de ocorrências que resultam em baixas taxas de mortalidade e fecundidade, denotando o quadro de envelhecimento populacional. Tal fato provocou uma mudança no 
perfil epidemiológico mundial, devido à melhoria da qualidade de vida, que conduziu a um crescimento das doenças crônicas não transmissíveis em detrimento das doenças infectocontagiosas ${ }^{(1)}$.

No Brasil, no período de 2011 a 2036, a estimativa de queda da taxa de fecundidade possibilitará que o país passe da fase jovem, com uma população com até $7 \%$ de pessoas com 60 anos ou mais, para o estágio envelhecido, em que contará com mais de 14\% de pessoas idosas. Em 2050, 38 milhões de brasileiros serão idosos e o grupo de idosos mais velhos, aqueles com 80 ou mais anos será o de maior crescimento ${ }^{(1)}$.

Nesse sentido, a Organização Mundial da Saúde alerta que embora, no momento, apenas o Japão possua uma proporção de idosos superior a 30\%, na segunda metade deste século, muitos países terão uma proporção similar. No Brasil, a população idosa corresponde hoje a cerca de 10 a 19\% da população total. Porém, no ano de 2050 ela representará algo em torno de 25 a 29\%. A adaptação do Brasil a essa situação deverá ser muito mais rápida do que a verificada em outros países ${ }^{(2)}$.

Contudo, a maioria dos idosos brasileiros (85\%) é atuante e autônoma contribuindo para o desenvolvimento socioeconômico, político e cultural do país. Cerca de $87 \%$ dos homens idosos chefiam famílias e mais da metade contribui com seus proventos para a renda dos lares ${ }^{(3)}$.

O aumento da longevidade associada a mudanças sociais e culturais faz também com que mais idosos busquem estilos de vida baseados em atividades recreativas, em ambientes amplos, muitos deles sem a devida acessibilidade, consequentemente tornando-os mais vulneráveis à ocorrência de acidentes ${ }^{(4)}$.

Dessa forma, embora o envelhecimento seja um processo natural, gradual e inevitável em todo ser vivo, nos humanos, as alterações fisiológicas características do idoso, associadas ou não a fatores secundários, podem contribuir para a ocorrência de determinados episódios que podem agravar a saúde da população idosa. Assim, certas situações, consideradas como causas externas, necessitam ser discutidas a fim de evitar prejuízos ao bem-estar desses indivíduos.

As causas externas de morbidade e mortalidade compreendem as lesões decorrentes de acidentes e de violências e representam um desafio às autoridades de saúde pública na atualidade, pois estão associadas a importante mortalidade e morbidade, além de grandes prejuízos financeiros e sociais ${ }^{(5)}$.

No Brasil, essas causas representam desde a década de 1980 a terceira causa de mortalidade e respondem pela sexta causa de internações, o que consiste em demanda significativa nos serviços de urgência e emergência ${ }^{(6)}$.

Os idosos, devido ao conjunto de fatores que acompanham o processo de envelhecimento, encontram-se expostos ao maior risco de acidentes domiciliares e dentre esses acidentes as quedas são os mais frequentes. Com isso, o domicílio que deveria ser um lugar seguro, torna-se muitas vezes um ambiente de $\operatorname{risco}^{(7)}$

Muitos desses idosos apresentam baixa acuidade visual e auditiva, assim como lentidão de reflexos e redução da força física. Alguns residirem sozinhos, mesmo possuindo dependência de cuidados, possibilitando maior risco de acidentes domiciliares ${ }^{(8)}$.

Pesquisa norte americana sobre prevenção de lesões não intencionais em ambiente doméstico que 
usou a pirâmide de impacto de saúde, afirma que essas lesões resultam de um conjunto de fatores comportamentais, físicos, estruturais, ambientais e sociais, destacou que a prevenção desses eventos representa um desafio ${ }^{(9)}$.

Estudo observacional de séries temporais que objetivou descrever a evolução das hospitalizações por causas externas no Brasil, no período de 2002 a 2011 mostrou que, em relação ao coeficiente de internação hospitalar por quedas, observou-se aumento em ambos os sexos, sendo o maior valor verificado entre os idosos, atingindo 6,3 internações/100 mil habitantes/ano ${ }^{(10)}$.

Entretanto, apesar do aumento na incidência desses agravos na população idosa, a produção cientifica que busca identificar fatores de risco, assim como as complicações e a mortalidade por essas causas nesse grupo etário ainda é incipiente. Destarte, o papel do enfermeiro como um ator social capaz de trabalhar para amenizar essas questões ser relevante, principalmente em relação à prevenção das ocorrências, por tratarse de uma população suscetível ${ }^{(11)}$.

Considerando os aspectos citados, foi definido como objeto de estudo os acidentes domésticos em idosos e fez-se o seguinte questionamento: quais os tipos de acidentes domésticos mais frequentes entre os idosos e a causa referida para sua ocorrência entre os idosos acidentados?

A produção científica abordando especificamente os acidentes domésticos com idosos no país ainda apresenta lacunas, requer analise mais detalhada das características e implicações desses acidentes no domicílio, considerando que a maioria dos estudos sobre o tema apresenta a generalidade das causas externas, embora se reconheça ser a queda um dos acidentes domésticos mais prevalentes nessa faixa etária. No entanto, a sociedade e os profissionais de saúde precisam conhecer os dados sobre os acidentes com idosos no domicílio, para que possam investir na prevenção e controle de sua ocorrência.

Os resultados da presente investigação poderão contribuir com reflexões acerca da necessidade de maior atenção ao idoso vitimado por acidente no domicílio, para que ao reconhecer as características específicas dessas pessoas, bem como os riscos que as cercam, seja possível desenvolver um cuidado efetivo e eficaz.

Neste contexto, objetivou-se analisar os acidentes domésticos em idosos atendidos em um hospital público de urgência.

\section{MÉTODOS}

Estudo descritivo de corte transversal realizado em um hospital de referência em urgência. A amostra por conveniência foi constituída por 83 idosos. Utilizou-se como técnica de coleta de dados a entrevista realizada por meio de um formulário. As variáveis definidas foram: sexo, idade, estado civil, escolaridade, com quem reside, renda mensal e origem da renda, tipo de acidente doméstico, presença de doença crônica, tipo de doença crônica, quantidade de medicamentos em uso, medicamentos utilizados diariamente, causa referida do acidente, realização de atividade no momento do acidente e tipo de atividade realizada no instante do acidente. Os dados do estudo foram coletados nos meses de junho e julho de 2014. Adotou-se 
como critérios de inclusão ter idade igual ou superior a 60 anos, de ambos os sexos, ter sido atendido no local de execução da pesquisa em decorrência de acidente doméstico e ser residente no estado em que a investigação foi realizada.

As informações obtidas foram codificadas em planilhas do aplicativo Microsoft Excel adotando-se um sistema de dupla digitação, com posterior correção, sendo então exportadas e analisadas pelo software Statistical Package for Social Sciences (SPSS). Foram examinadas associações estatísticas utilizando o Teste Exato de Fisher, produtor de menor erro em amostras pequenas, realizado no programa StatXact-3, sendo a hipótese de associação aceita quando o p encontrado foi menor ou igual a 0,05.

Os participantes expressaram sua concordância por meio da assinatura do Termo de Consentimento Livre e Esclarecido ou, no caso dos analfabetos, pela impressão digital no documento. A pesquisa obedeceu às diretrizes das normas brasileiras para pesquisas com seres humanos. O projeto foi aprovado por meio do parecer de no 09/14 da Comissão de Ética em Pesquisa do Hospital de Urgência e do parecer no 675.125 do Comitê de Ética em Pesquisa da Universidade Federal do Piauí e CAAE no 31174514.0.0000.5214.

\section{RESULTADOS}

A prevalência de acidentes domésticos no período de coleta foi $6,4 \%$. No que se refere ao perfil sociodemográfico dos idosos vitimados constata-se na Tabela 1 que houve predomínio das mulheres mais velhas, viúvas, não alfabetizadas que moram com a família e recebem entre um e dois salários mínimos.

A Tabela 2 demonstra que a maioria dos participantes encontrava-se internada em decorrência de queda no domicílio, correspondendo a $84,4 \%$. Além disso, a causa mais referida foi o descuido na observação do ambiente com 38 respostas positivas $(45,8 \%)$, seguida por valores semelhantes entre deficiência na locomoção e outras causas, ambas com $15,7 \%$ dos entrevistados, respectivamente, sendo que as últimas incluem hipotensão postural, vertigens e desmaios.

Na Tabela 3 observa-se que ao relacionar-se o tipo de acidente e sexo, há um predomínio feminino nas quedas, representado por 56 idosas $(67,6 \%)$, sendo que a maior parte refere ter caído da própria altura, com um número de 39 participantes (47,2\%). Os ferimentos ocupam a segunda posição, embora evidenciem diferença acentuada comparativamente ao primeiro lugar, com um total de 8 idosos $(9,6 \%)$.

Ao analisar-se, na Tabela 4, a relação entre a ocorrência de queda, a existência de doença crônica, o uso contínuo de medicamentos e a realização de atividades no momento do acidente (domésticas ou de higiene), constatou-se a ausência de associação entre as variáveis, visto que os valores de $p>0,05$. 
Tabela 1: Distribuição dos idosos envolvidos em acidentes domésticos atendidos em um hospital de referência em urgência, segundo aspectos sociodemográficos. Teresina, PI, Brasil, 2014.

\begin{tabular}{|c|c|c|c|c|c|}
\hline Variáveis & Média (Desvio Padrão) & Mediana & Variação Observada & Distribuição em categorias & n (\%) \\
\hline \multirow{4}{*}{ Faixa Etária (anos) } & $76,7(9,8)$ & 78 & {$[60 ; 96]$} & $60-69$ & $22(26,6)$ \\
\hline & & & & $70-79$ & $26(31,3)$ \\
\hline & & & & $80-89$ & $27(32,5)$ \\
\hline & & & & $90-96$ & $8(9,6)$ \\
\hline \multirow{2}{*}{ Sexo } & & & & Feminino & $64(77,1)$ \\
\hline & & & & Masculino & $19(22,9)$ \\
\hline \multirow{4}{*}{ Estado Civil } & & & & Solteiro & $10(12,0)$ \\
\hline & & & & Casado & $32(38,6)$ \\
\hline & & & & Divorciado & $4(4,8)$ \\
\hline & & & & Viúvo & $37(44,6)$ \\
\hline \multirow{4}{*}{ Escolaridade } & & & & Analfabeto & $50(60,2)$ \\
\hline & & & & Fundamental & $27(32,6)$ \\
\hline & & & & Médio & $5(6,0)$ \\
\hline & & & & Superior & $1(1,2)$ \\
\hline \multirow{5}{*}{ Com quem mora } & & & & Cônjuge e outros familiares & $61(73,5)$ \\
\hline & & & & Com o cônjuge & $12(14,5)$ \\
\hline & & & & Sozinho & $7(8,4)$ \\
\hline & & & & ILP & $1(1,2)$ \\
\hline & & & & Outro & $2(2,4)$ \\
\hline \multirow{4}{*}{ Renda mensal } & & & & Menos 1SM & $7(8,4)$ \\
\hline & & & & 1 a 2 SM & $73(88,0)$ \\
\hline & & & & 3 a 5 SM & $2(2,4)$ \\
\hline & & & & Ignorado & $1(1,2)$ \\
\hline \multirow{6}{*}{ Origem renda mensal } & & & & Aposentadoria & $70(84,4)$ \\
\hline & & & & Pensão & $5(6,0)$ \\
\hline & & & & Mais de fonte & $4(4,8)$ \\
\hline & & & & Doação & $1(1,2)$ \\
\hline & & & & Ignorado & $1(1,2)$ \\
\hline & & & & Outro & $2(2,4)$ \\
\hline
\end{tabular}

Tabela 2: Distribuição dos idosos envolvidos em acidente domésticos de acordo com o tipo de acidente, segundo a causa referida pelo idoso. Teresina, PI, Brasil, 2014.

\begin{tabular}{|c|c|c|c|c|c|c|c|c|c|c|}
\hline \multirow{2}{*}{ Variáveis } & \multicolumn{2}{|c|}{ Queda } & \multicolumn{2}{|c|}{ Ferimento } & \multicolumn{2}{|c|}{ Queimadura } & \multicolumn{2}{|c|}{ Intoxicação } & \multicolumn{2}{|c|}{ Total } \\
\hline & $n$ & $\%$ & $n$ & $\%$ & $n$ & $\%$ & $\mathrm{n}$ & $\%$ & $n$ & $\%$ \\
\hline Descuido na observação do ambiente & 33 & 39,8 & 4 & 4,8 & - & - & 1 & 1,2 & 38 & 45,8 \\
\hline Deficiência na locomoção & 12 & 14,5 & 1 & 1,2 & - & - & - & - & 13 & 15,7 \\
\hline Descuido ao realizar atividade & 7 & 8,4 & 3 & 3,6 & 1 & 1,2 & - & - & 11 & 13,2 \\
\hline Objetos mal posicionados & 4 & 4,8 & - & - & - & - & - & - & 4 & 4,8 \\
\hline Deficiência na visão & 4 & 4,8 & - & - & - & - & - & - & 4 & 4,8 \\
\hline Outro & 10 & 12,1 & - & - & 2 & 2,4 & 1 & 1,2 & 13 & 15,7 \\
\hline Total & 70 & 84,4 & 8 & 9,6 & 3 & 3,6 & 2 & 2,4 & 83 & 100 \\
\hline
\end{tabular}


Tabela 3: Distribuição dos idosos envolvidos em acidentes domésticos de acordo com o sexo, segundo o tipo de acidente. Teresina, PI, Brasil, 2014.

\begin{tabular}{|c|c|c|c|c|c|c|}
\hline \multirow{3}{*}{ Variáveis } & \multicolumn{4}{|c|}{ Sexo } & \multirow{2}{*}{\multicolumn{2}{|c|}{ Total }} \\
\hline & \multicolumn{2}{|c|}{ Feminino } & \multicolumn{2}{|c|}{ Masculino } & & \\
\hline & $\mathbf{n}$ & $\%$ & $\mathbf{n}$ & $\%$ & $\mathbf{n}$ & $\%$ \\
\hline \multicolumn{7}{|l|}{ Queda } \\
\hline Própria Altura & 39 & 47,2 & 7 & 8,4 & 46 & 55,6 \\
\hline Cadeira/Poltrona & 3 & 3,6 & 3 & 3,6 & 6 & 7,2 \\
\hline Cama & 5 & 6,0 & - & - & 5 & 6,0 \\
\hline Cadeira de Banho/Vaso Sanitário & 1 & 1,2 & 1 & 1,2 & 2 & 2,4 \\
\hline Escada & 1 & 1,2 & - & - & 1 & 1,2 \\
\hline Outro & 7 & 8,4 & 3 & 3,6 & 10 & 12,0 \\
\hline Total & 56 & 67,6 & 14 & 16,8 & 70 & 84,4 \\
\hline \multicolumn{7}{|l|}{ Queimadura } \\
\hline Líquido Quente & - & - & 2 & 2,4 & 2 & 2,4 \\
\hline Produto Químico & 1 & 1,2 & - & - & 1 & 1,2 \\
\hline Total & 1 & 1,2 & 2 & 2,4 & 3 & 3,6 \\
\hline \multicolumn{7}{|l|}{ Intoxicação } \\
\hline Alimento & 1 & 1,2 & - & - & 1 & 1,2 \\
\hline Derivado de Petróleo & 1 & 1,2 & - & - & 1 & 1,2 \\
\hline Total & 2 & 2,4 & - & - & 2 & 2,4 \\
\hline \multicolumn{7}{|l|}{ Ferimento } \\
\hline Ferro & 1 & 1,2 & - & - & 1 & 1,2 \\
\hline Estilete & - & - & 1 & 1,2 & 1 & 1,2 \\
\hline Outro & 4 & 4,8 & 2 & 2,4 & 6 & 7,2 \\
\hline Total & 5 & 6,0 & 3 & 3,6 & 8 & 9,6 \\
\hline Total Geral & 64 & 77,2 & 19 & 22,8 & 83 & 100 \\
\hline
\end{tabular}

Tabela 4: Relação entre a ocorrência de quedas em idosos e a existência de doença crônica, utilização de medicamentos de uso contínuo e a realização de atividades domésticas e de higiene. Teresina, PI, Brasil, 2014.

\begin{tabular}{|c|c|c|c|c|}
\hline Variáveis & \multicolumn{2}{|c|}{ Quedas } & \multirow[t]{2}{*}{ Fisher } & \multirow[t]{2}{*}{$p$-value } \\
\hline Doença Crônica & Sim & Não & & \\
\hline Sim & 53 & 10 & 0,008 & 1,00 \\
\hline Não & 17 & 03 & & \\
\hline Total & & 83 & & \\
\hline \multicolumn{5}{|l|}{ Medicamento } \\
\hline Sim & 53 & 10 & 0,014 & 1,00 \\
\hline Não & 17 & 03 & & \\
\hline Total & & 83 & & \\
\hline \multicolumn{5}{|l|}{ Atividade doméstica } \\
\hline Sim & 08 & 03 & 1,294 & 0,3663 \\
\hline Não & 62 & 10 & & \\
\hline Total & & 83 & & \\
\hline \multicolumn{5}{|l|}{ Atividade de Higiene } \\
\hline Sim & 04 & 02 & 1,525 & 0,2358 \\
\hline Não & 66 & 11 & & \\
\hline Total & & 83 & & \\
\hline
\end{tabular}

\section{DISCUSSÃO}

Ao se considerar os dados sociodemográficos encontrados, observa-se que houve predomínio das mulheres entre os entrevistados, caracterizando a feminização da velhice, em decorrência do maior índice de mortalidade masculina, tanto por causas violentas quanto pela baixa procura pelos serviços de saúde. Isto 
se dá muitas vezes por questões culturais, pois muitos homens ainda veem a procura pelo cuidado como uma prática exclusivamente feminina ${ }^{(12)}$.

Estudo realizado no Paraná com 324 idosos vitimados por causas externas encontrou resultado semelhante, observando que $55,2 \%$ dos idosos estudados eram mulheres, na faixa etária de 60 a 65 anos $(26,2 \%)$, seguida de 80 anos ou mais (24,6\%). A maioria das ocorrências aconteceram nos domicílios (52,7\%), sendo as quedas de mesmo nível as causas mais frequentes $(57,1 \%)^{(13)}$. Pesquisa desenvolvida no norte da Suécia para estudar a epidemiologia das lesões não intencionais em uma população de 21.000 idosos verificou que a taxa de acidentes foi maior entre as mulheres com 75 anos e mais, constatando que as quedas ocorridas nas instituições de longa permanência causaram as lesões mais graves ${ }^{(14)}$.

Investigação que identificou as causas e consequências de quedas em idosos no domicílio constatou necessidade de estudos com análise mais profunda acerca da questão de gênero, ocorrência de quedas e atividades cotidianas, para que os programas de prevenção possam considerar essa variável nas orientações sobre casa segura para o idoso ${ }^{(7)}$.

Outra informação relevante é a existência da alta taxa de idosos analfabetos, apesar da implantação de programas governamentais de combate ao analfabetismo, como o Brasil Alfabetizado. Investigações desenvolvidas com idosos nas regiões sudeste e sul do país, que objetivaram estimar a prevalência de quedas e verificar os fatores associados ao medo de queda recorrente, encontraram resultados diferentes da presente investigação, verificando que a maior parte dos participantes possuíam entre um a quatro anos de estudo $^{(15-16)}$. Acredita-se que esse fato seja decorrente de uma situação educacional existente no nordeste brasileiro, sendo fundamental permanecer ainda hoje com programas de alfabetização para adultos.

Fatores socioeconômicos como o baixo nível de escolaridade, a pobreza e alguma privação podem aumentar a exposição a riscos ambientais, assim como influenciam também na exposição a riscos de acidentes específicos, na forma de habitação insegura, acesso inadequado a cuidados preventivos de saúde e falta de acesso a dispositivos de segurança, entre outros ${ }^{(9)}$.

Os resultados do presente estudo mostraram ainda que a maioria dos acidentados morava com o cônjuge e/ou algum familiar, era aposentada e recebia entre um e dois salários mínimos. Segundo dados dos últimos censos realizados pelo Instituto Brasileiro de Geografia e Estatística, idosos com esse perfil são geralmente responsáveis pelos domicílios, tanto financeiramente quanto na organização do lar. Estes números mostram também que muitos líderes de famílias moram apenas com filhos, netos ou outra pessoa da família ${ }^{(17)}$, caracterizando o quadro da viuvez.

Pesquisa realizada em um hospital público de Paranamirim com o objetivo de construir um perfil dos atendimentos por causas externas, mesmo não havendo sido delimitada uma faixa etária específica, demonstrou que as quedas ocupavam a primeira posição, com $29 \%$ dos casos, os acidentes domésticos ocuparam a quarta posição, com $16,5 \%$, e com menores percentuais, queimadura $(3,24 \%)$ e intoxicação exógena $(0,15 \%)^{(6)}$. Ainda que as duas últimas causas não tenham ocorrido somente no domicílio, verifica-se uma semelhança com o presente estudo, em que as queimaduras constituíram 3,6\%, e intoxicações $2,4 \%$. 
A preponderância da queda, dentre os acidentes domésticos, encontrada na presente pesquisa assemelha-se a um estudo realizado em um pronto socorro situado no Rio Grande do Sul sobre as causas externas envolvendo idosos. Naquele estudo, a queda representou $48 \%$ dos casos, sendo possível verificar na mesma investigação que os cortes constituíram $4,1 \%$ do total, em conformidade com a diferença encontrada neste estudo, mesmo que o local de ocorrência do acidente não tenha sido especificado no citado trabalho ${ }^{(18)}$.

Embora uma diferença significante tenha ocorrido entre a queda e os outros acidentes domésticos, eles são relevantes no que concerne à conservação da saúde do idoso. Os ferimentos, caso não sejam devidamente tratados, podem evoluir para infeç̧ões e mesmo afecções mais graves, inclusive em idosos diabéticos, propiciando uma hospitalização prolongada. O mesmo acontece com as queimaduras, que dependendo do grau e extensão, podem influenciar sobremaneira na qualidade de vida do paciente, na medida em que representa um quadro clínico facilmente gerador de complicações. Por outro lado, as intoxicações também constituem uma causa de prejuízos ao bem-estar dos idosos, principalmente em decorrência da polifarmácia, fenômeno comum a esse grupo.

Estudo norte americano realizou observação em 603 casas com objetivo de descrever a prevalência de medidas preventivas de segurança em habitações com idosos e descrever o conhecimento de temas de segurança por esses indivíduos, concluindo que é necessário esforço para melhorar o conhecimento e promover benefícios de salvamento com medidas contra ferimento para que a população idosa possa ser adequadamente protegida. Recomendou ainda que os profissionais e pesquisadores devem continuar a desenvolver e avaliar programas que visem reduzir os riscos de lesão em casas com idosos para que eles possam envelhecer com segurança ${ }^{(19)}$.

As estratégias de cuidados profissionais para a prevenção de quedas em idosos são fundamentais. Incluem orientações de cuidados por meio de ações de conscientização dos idosos e familiares, envolvendo informações sobre riscos, mudanças no estilo de vida e modificações ambientais. Isso requer capacitação dos profissionais de saúde em relação à prevenção e o gerenciamento de quedas ${ }^{(13)}$. Destaca-se que o descuido na observação do ambiente, principal motivo referido pelos entrevistados neste estudo, demonstra o quão relevante deve ser a atenção dada ao meio físico em que eles vivem.

Nesse sentido, revisão da literatura realizada nos Estados Unidos da América afirma que as causas de quedas em pessoas idosas são múltiplas e incluem fatores como déficits físicos e/ou visuais, declínio na função cognitiva, redução da função proprioceptiva e atraso no tempo de resposta, já conhecidos por afetarem equilíbrio, marcha, postura e movimento. Os medicamentos também podem influenciar para ocorrência desse evento devido aos efeitos secundários, tais como tonturas e/ou alterações de humor. Igualmente, iluminação deficiente contribui também para essa ocorrência. Todos esses fatores afetam, invariavelmente, desempenho e função ocupacional dos idosos ${ }^{(20)}$.

Ao se considerar as diferentes causas das quedas, outro fator que também pode contribuir para a ocorrência desse tipo de acidente pode ser a diminuição dos hormônios produzidos pelas mulheres devido à 
chegada da menopausa, fazendo com que elas se tornem mais frágeis fisicamente e mais propensa a doenças como osteoporose e artrose $\mathrm{e}^{(12,17-18)}$.

Outro aspecto a ser também levantado, se deve ao fato da aposentadoria no sexo feminino ocorrer mais cedo do que no sexo masculino. Consequentemente, as mulheres passam mais tempo dentro de suas residências realizando atividades domésticas ${ }^{(7,13)}$.

Porém independente da causa, os acidentes podem trazer ainda consequências econômicas aos idosos e a sua família, pois muitas vezes as lesões decorrentes do acidente, requerem reabilitação posterior.

Estudo transversal desenvolvido com 389 idosos para identificar as causas e consequências de quedas de idosos em domicílio alerta sobre o número de fraturas decorrentes das quedas e a diversidade de possibilidades para ocorrência desse evento. Destaca a necessidade de mais estudos sobre a temática, para investigar o vínculo com as atividades cotidianas ${ }^{(7)}$.

Um grande número de entrevistados sofreu queda da própria altura, informação corroborada por estudo transversal realizado em João Pessoa, em que 90,5\% dos idosos tiveram o mesmo tipo de queda ${ }^{(17)}$. Esse dado é preocupante, pois a maioria deles não referiu estar realizando nenhuma atividade no momento do acidente. Infere-se que este fato se deve às mudanças corporais sofridas pelas pessoas idosas já que, com a diminuição da deambulação, o indivíduo vai perdendo massa e força muscular, tornando-se mais frágil e propenso a quedas ${ }^{(17-18)}$.

Revisão sistemática sobre o envolvimento de fatores ambientais nas quedas em idosos vivendo na comunidade concluiu que o local de ocorrência das quedas sofre influência de fatores intrínsecos, pois verificou que idosos ativos fisicamente tendem a cair em ambientes externos, ao passo que idosos com comprometimento funcional caem no próprio domicílio ${ }^{(21)}$.

Investigação realizada em oito cidades europeias no contexto de um projeto da Organização Mundial da Saúde que analisou as condições de habitação e o estado de saúde de 8.519 pessoas, verificou que as condições de moradia inadequadas, que não atendiam às condições dos residentes representavam um fator de risco direto para acidentes e lesões, sendo a queda um problema sério para os idosos, com alta incidência na faixa entre 60 e 79 anos, e duas vezes maior entre aqueles com idade superior a 80 anos $^{(22)}$.

Pesquisa desenvolvida com 108 idosos na cidade de Campinas, em que 77,8\% e 67,7\% dos entrevistados, respectivamente, possuíam patologias e faziam uso contínuo de medicação, observou que dentre os idosos que sofreram queda, ocorreu predomínio daqueles que possuíam doenças crônicas e utilizavam medicamentos de uso contínuo ${ }^{(23)}$.

A ausência de associação entre a ocorrência de quedas e a presença de doença crônica assemelhouse a estudo realizado com idosos não institucionalizados, de 80 anos ou mais em uma área da Estratégia de Saúde da Família na Bahia, em que a ocorrência de quedas não foi associada à presença de doenças crônicas ${ }^{(24)}$. Tal dado pode ser explicado pelo fato de todos os idosos ingerirem os medicamentos apropriados à sua condição clínica, evitando manifestações indesejáveis.

Por outro lado, as medicações de uso contínuo podem ocasionar efeitos colaterais que influenciam 
sobremaneira a ocorrência de quedas. Conforme uma pesquisa bibliográfica realizada para analisar os fatores de risco de quedas em idosos, verificou-se quantidade considerável de estudos associando essa característica à possibilidade desse acidente ${ }^{(25)}$.

Apesar de não terem sido encontradas publicações que corroborassem com o achado deste estudo, tal dado apresenta conformidade com os outros resultados encontrados, na medida em que os fatores fisiológicos constituíram menor circunstância responsável pela ocorrência dos acidentes. Além disso, aspectos como classes medicamentosas e quantidade de medicamentos devem ser considerados.

A não associação entre a ocorrência de quedas e a realização de atividades no instante do acidente pode estar relacionada a fato de que a maioria dos indivíduos entrevistados possui idade avançada, ou seja, maior dependência, necessitando de auxílio para a realização de seus afazeres, fato também corroborado pela predominância de idosos que moram com familiares, estando assim, teoricamente, mais assistidos.

\section{CONCLUSÃO}

Os resultados encontrados na amostra estudada evidenciaram uma prevalência de acidentes domésticos de $6,4 \%$, sendo a queda da própria altura o acidente doméstico de maior frequência entre os idosos estudados, os quais referiram o descuido na observação do ambiente como a principal causa das ocorrências.

Porém, certas peculiaridades como a ausência de associação entre a ocorrência de quedas, presença de doença crônica, medicação de uso contínuo e realização de atividade no momento do acidente também foram observadas.

Tal quadro demonstra que o ambiente no qual o idoso está inserido deve ser adequado às suas necessidades, na medida do possível, em conformidade com as condições financeiras da família. A presença familiar também é importante a fim de auxiliar, especialmente, os idosos que apresentam maior dependência, tanto na realização das atividades cotidianas, quanto no âmbito da saúde.

A alta representatividade que esses acidentes ocupam nos índices de morbidade e mortalidade entre os idosos requer maior vigilância e investigação detalhada por parte dos profissionais de saúde, tanto dentro dos hospitais, por intermédio da anamnese completa, quanto em unidades básicas, por meio das consultas mensais e visitas domiciliares, para fazer a diferença na notificação dessas ocorrências e traçar o perfil epidemiológico característico da população idosa afetada, possibilitando assim a orientação acerca da prevenção e controle desses eventos indesejáveis.

Os resultados do estudo mostraram também que é preciso investir em estratégias específicas para prevenir e controlar acidentes domésticos com idosos, uma vez que os próprios idosos acreditam que as ocorrências poderiam ser evitadas, ao afirmarem que o acidente ocorreu por descuido na observação do ambiente.

Portanto, é importante que os profissionais reconheçam a necessidade de uma formação permanente sobre prevenção de acidentes com idosos, desenvolvam um cuidado multiprofissional com avaliação 
funcional ampla desses indivíduos a fim de identificar precocemente as deficiências por eles apresentadas, assim como as limitações do ambiente que os cerca para orientar familiares e cuidadores, como também possibilitar que cada profissional atue em suas especificidades colaborando no planejamento de intervenções integradas, com garantia de atendimento das necessidades de cuidado e disponibilidade de tecnologias necessárias para auxílio e superação das dependências identificadas.

Apesar deste estudo trazer a realidade de uma situação local, seus achados mostram que o desenvolvimento de investigações no contexto da atenção primária poderiam mapear melhor as informações sobre a morbidade pelos acidentes domésticos e possibilitariam melhorar o planejamento de cuidados na comunidade.

\section{REFERÊNCIAS}

1. Chaimowicz F. Saúde do Idoso. 2. ed. Belo Horizonte: NESCON UFMG; 2013.

2. Organización Mundial de la Salud. Informe mundial sobre el envejecimiento y la salud. [Internet]. Ginebra: Organización Mundial de la Salud; 2015 [acesso em: 08 abr 2016]. Disponível em: http://apps.who.int/iris/bitstream/10665/186466/1/9789240694873_spa.pdf.

3. Minayo MCS. O envelhecimento da população brasileira e os desafios para o setor saúde. Cad. Saúde Pública. 2012;28(2):208-209.

4. Degani GC, Júnior GAP, Rodrigues RAP, Luchesi BM, Marques S. Idosos vítimas de trauma: doenças preexistentes, medicamentos em uso no domicílio e índices de trauma. Rev. Bras. Enferm. 2014; 67(5): 759-765.

5. Mascarenhas MDM, Monteiro RA, Sá NNB, Gonzaga LAA, Neves ACM, Silva MMA, Malta DC. Epidemiologia das causas externas no Brasil: morbidade por acidentes e violências In: Ministério da Saúde (BR). Saúde Brasil 2010: uma análise da situação de saúde e de evidências selecionadas de impacto de ações de vigilância em saúde. Brasília: Ministério da Saúde; 2011. p. 203-24.

6. Lima MVF, Silva RLP, Albuquerque NMG, Oliveira JSA, Cavalcante CAA, Macêdo MLAF. Perfil dos atendimentos por causas externas em hospital público. Rev Rene. 2012; 13(1): 36-43.

7. Ferretti F, Lunardi D, Bruschi L. Causas e consequências de quedas de idosos em domicílio. Fisioter. Mov. 2013; 26(4): 753-62.

8. Serra MC, Guimarães Junior LM, Sperandio A, Stoffel C, Zocrato K, Neves L, Lopes O. Queimadura em pacientes da terceira idade: epidemiologia de 2001 a 2010. Rev. Bras. Queimaduras. [Internet]. 2011; [acesso em: 27 abri 2016]; 10(4): 111-3. Disponível em: file:///C:/Users/Usuario/Downloads/v10n4a03.pdf.

9. Mack KA, Liller KD, Baldwin G, Sleet D. Preventing Unintentional Injuries in the Home Using the Health Impact Pyramid. Health educ. behav. 2015; 42 (1S): 115-122.

10. Mascarenhas MDM, Barros MBA. Evolução das internações hospitalares por causas externas no sistema público de saúde - Brasil, 2002 a 2011. Epidemiol. Serv. Saúde. 2015; 24(1): 19-29.

11. Rodrigues J, Ciosak SI. Idosos vítimas de trauma: análise de fatores de risco. Rev Esc Enferm USP. 2012; 46(6):14005.

12. Furtado LFV, Araújo PM, Soares FVS, Brito VM, Sousa LG, Melo ACL, et al. Epidemiologia do envelhecimento: dinamização, problemas e consequências. Revista Kairós Gerontologia. [Internet] 2012; [acesso em: 20 mar 2016 ];15

(1): 55-69. Disponível em: http://revistas.pucsp.br/index.php/kairos/article/view/13106/9635.

13. Grden CRB, Sousa JAV, Lenardt MH, Pesck RM, Seima MD, Borges PKO. Caracterização de idosos vítimas de acidentes por causas externas. Cogitare Enferm. 2014; 19(3): 506-13.

14. Saveman BI, Björnstig U. Unintentional injuries among older adults in northern Sweden: a one-year populationbased study. Scand J Caring Sci. 2011; 25 (1): 185-193.

15. Fhon JRS, Fabrício-Wehbe SCC, Vendruscolo TRP, Stackfleth R, Marques S, Rodrigues RAP. Quedas em idosos e sua relação com a capacidade funcional. Rev. Latino-Am. Enfermagem. 2012; 20(5): 944-953.

16. Antes DL, Schneider IJC, Benedetti TRB, D'Orsi E. Medo de queda recorrente e fatores associados em idosos de Florianópolis, Santa Catarina, Brasil. Cad. Saúde Pública. 2013; 29(4):758-768. 
17. Fernandes MGM, Barbosa KTF, Oliveira FMRL, Rodrigues MMD, Santos KFO. Risco de quedas evidenciado por idosos atendidos num ambulatório de geriatria. Rev. Eletr. Enf. [Internet]. 2014 [acesso em: 10 fev 2015]; 16(2):297303. Disponível em: http://dx.doi.org/10.5216/ree.v16i2.20542.

18. Maag SB, Lange CL, Linck CL, Gigante DP, Pereira PM, Quadros LCM. Causas externas envolvendo idosos atendidos em um pronto socorro. Rev Enferm UFPE [Internet]. 2013; [acesso em: 10 fev 2015]; 7(8):5274-9. Disponível em: 10.5205/r euol.3452-28790-4-ED.0708201315.

19. Shields WC, Perry EC, Szanton SL, Andrews MR, Stepnitz RL, McDonald EM, Gielen AC. Knowledge and injury prevention practices in homes of older adults. Geriatric Nursing. 2013; 34(1):19-24.

20. Paul S, Liu Y. Inadequate light levels and their effect on falls and daily activities of community dwelling older adults: a review of Literature. New Zealand Journal of Occupational Therapy. 2012; 59(2):39-42.

21. Oliveira AS, Trevizan PF, Bestetti MLT, Melo RC. Fatores ambientais e risco de quedas em idosos: revisão sistemática. Rev. Bras. Geriatr. Gerontol. 2014; 17(3): 637-645.

22. Braubach $M$, Power $A$. Housing conditions and risk: reporting on a european study of housing quality and risk of accidents for older people. Journal of Housing for the Elderly; 2011; 25 (3): 288-305.

23. Lima R S, Campos M L P. Perfil do idoso vítima de trauma atendido em uma Unidade de Urgência e Emergência. Rev Esc Enferm USP. 2011; 45(3):659-64.

24. Brito TA, Fernandes MH, Coqueiro RS, Jesus CS. Quedas e capacidade funcional em idosos longevos residentes em comunidade. Texto Contexto Enferm. 2013; 22(1): 43-51.

25. Costa ICP, Lopes MEL, Andrade CG, Souto MC, Costa KC, Zaccara AAL. Fatores de risco de quedas em idosos: produção científica em periódicos online no âmbito da saúde. Rev bras ciênc saúde [Internet] 2012; [acesso em: 10 fev 2015]; 16(3): 445-452. Disponível em: http://periodicos.ufpb.br/ojs/index.php/rbcs/article/view/12882/7888. 